\title{
An Experience on Normothermic Beating Heart Pulmonary Thromboendarterectomy
}

\author{
Akshay Kumar Bisoi1, Pradeep Ramakrishnan1*, Sandeep Chauhan², Manoj Kumar Sahu1, \\ Naveen Chander Chandrasekharan ${ }^{1}$
}

\author{
${ }^{1}$ Department of CTVS, C N Centre, All India Institute of Medical Sciences, New Delhi, India \\ ${ }^{2}$ Department of Cardiac Anaesthesiology, C N Centre, All India Institute of Medical Sciences, New Delhi, India \\ Email: * pradeep.r.reddy85@gmail.com
}

How to cite this paper: Bisoi, A.K., Ramakrishnan, P., Chauhan, S., Sahu, M.K. and Chandrasekharan, N.C. (2019) An Experience on Normothermic Beating Heart Pulmonary Thromboendarterectomy. World Journal of Cardiovascular Surgery, 9, 1-13. https://doi.org/10.4236/wjcs.2019.91001

Received: December 18, 2018

Accepted: January 28, 2019

Published: January 31, 2019

Copyright $\odot 2019$ by author(s) and Scientific Research Publishing Inc. This work is licensed under the Creative Commons Attribution International License (CC BY 4.0).

http://creativecommons.org/licenses/by/4.0/

\begin{abstract}
Objective: To study the outcome of normothermic beating heart pulmonary thromboendarterectomy (PTE) performed in patients with chronic pulmonary thromboembolism. Methodology: A retrospective analysis of 15 patients who underwent PTE under normothermic beating heart cardiopulmonary bypass from July 2009 to September 2018 was done. The patients were followed up with clinical assessment, transthoracic echocardiography and computerized tomographicangiography (CTA) for 1 month to 82 months. Results: Data were analysed for 15 consecutive patients. Mean age of the patients was 35.28 years and 10 patients were male. Pre-operatively 6 (40\%) patients presented with New York Heart Association (NYHA) class II dyspnoea on exertion symptomatology, 7 (47\%) were in NHYA class III, and $2(13 \%)$ were in NYHA class IV. The preoperative and postoperative mean pulmonary artery pressures (mPAP) $(\mathrm{mmHg})$ were $36.50 \pm 11.3$ and $20.21 \pm 7.19$, the systolic PAPs $(\mathrm{mmHg})$ were $73.35 \pm 14.12$ and $35.21 \pm 7.89$ and the diastolic PAPs (mmHg) were $19.28 \pm 8.60$ and $12.85 \pm 7.26$ respectively. 2 (13\%) patients had Jamieson's type I and 12 (87\%) had Jamieson type II disease. One patient $(7 \%)$ expired on $9^{\text {th }}$ postoperative day. All the patients had improved pulmonary gas exchange and did not require oxygen supplementation from $5^{\text {th }}$ postoperative day; symptoms improved to NYHA class I \& II in $12(80 \%)$ \& 3 (20\%) of patients respectively. There was no reperfusion pulmonary edema or any neurologic complications. Postoperative echocardiogram showed improved right ventricular function and Computerised Tomographic Angiogram showed completeness of the procedure. Conclusion: Pulmonary thromboendarterectomy under normothermic beating heart cardiopulmonary bypass has good immediate postoperative results with significant progressive improvement in hemodynamics and quality of life during the course of follow-up. The results were not only comparable to those of the procedure done
\end{abstract}


under deep hypothermic circulatory arrest by other centres but also without its associated adverse events. This technique requires more expertise but gives equivalent good results in immediate and short- to mid-term follow-up with less morbidity than the standard procedure, but it requires long-term follow-up to substantiate the evidence.

\section{Keywords}

Pulmonary Thromboendarterectomy, Normothermic, Chronic Thromboembolic Pulmonary Hypertension (CTEPH), Beating Heart

\section{Introduction}

Chronic thromboembolic pulmonary hypertension (CTEPH) is a debilitating condition with an incidence rate of $3.8 \%$ of all cases of pulmonary embolism [1]. CTEPH is a sequelae of single or multiple episodes of acute pulmonary embolism leading to branch pulmonary artery obstruction with incomplete resolution, fibrosis, organization and endothelisation of the thrombus [2]. This process eventually leads to increased pulmonary vascular resistance (PVR), pulmonary hypertension (PHTN) and right heart failure. In general, symptoms develop late and dyspnoea on exertion is the most frequent presenting symptom. Less commonly, exercise intolerance, pleuritic chest pain, palpitations and hemoptysis can occur [3]. Chest x-ray and electrocardiogram (ECG) can suggest features of PHTN and right ventricular hypertrophy (RVH), but are not diagnostic. Transthoracic echocardiography (TTE) is the initial diagnostic modality and computerised tomographic angiogram (CTA) is the investigation of choice nowadays for diagnosing CTEPH [4]. Next investigation which deserves mention is right heart catheterisation, which can provide hemodynamic as well as anatomic details like RV pressures, PA pressures and pulmonary vascular resistance (PVR). But our strategy is to do right heart catheterisation only in situations where there is diagnostic dilemma or there is suspicion of coronary artery disease. Pulmonary thromboendarterectomy (PTE) under deep hypothermic circulatory arrest (DHCA) is the current standard treatment. This technique has the advantage of bloodless surgical field allowing a complete removal of the thrombus from the pulmonary vascular bed up to the sublobar levels. Even though DHCA gives the advantage of good surgical field, it is associated with dangers of potential neurological complications, as well as kidney, liver and spinal cord ischemia.

To avoid the above complications of DHCA, several attempts have been made by many groups worldwide. Masuda et al. performed PTE under DHCA with anterograde cerebral perfusion by selective cannulation of axillary or carotid arteries for brain protection in a series of 4 patients and they were able to safely extend the circulatory arrest time without any brain damage with complete thromboendarterectomy [5]. Hagl et al. performed PTE under total CPB with selective antegrade cerebral perfusion in 30 patients under moderate hypother- 
mia $\left(28^{\circ} \mathrm{C}\right)$ and by occlusion of Descending Thoracic Aorta by inflatable balloon catheter inserted just near the aortic inflow cannula, to prevent the bronchial back bleed. Seven patients had mild to moderate lung reperfusion injury, one patient had transient neurologic dysfunction and three patients died, out of whom two were due to multiorgan dysfunction and one was due to persistent pulmonary hypertension. This study has demonstrated the effective complete removal of thrombus with a good neurological outcome, but with significant lung reperfusion injury [6]. Mikus et al. in a series of eight patients performed PTE by double venting of the left heart (placing a vent in left atrium and left ventricle) and connected with vacuum device to prevent bronchial back bleeding and left ventricle distension. The temperature was lowered till $26^{\circ} \mathrm{C}$ so that DHCA was avoided and there was no neurologic complication in this series. This method is applicable especially to Jamieson's type I and II disease [7]. In all the above series, PTE was done under some degree of hypothermia and cardiac arrest with or without DHCA with antegrade cerebral perfusion. In a series of four patients by Zund et al., PTE was done under normothermic beating heart cardiopulmonary bypass (CPB) if interatrial septum was intact or if a patent's foramen ovale was present under electrically induced ventricular fibrillation with no adverse neurologic outcome. For a better exposure, superior vena cava was transected and reanastomosed after the PTE was completed [8].

This study is an attempt to perform PTE under normothermic beating heart $\mathrm{CPB}$, with no less accurate \& total removal of thrombus and without subjecting the patients into more potential complications of DHCA and longer CPB. This retrospective study was aimed at monitoring the safety \& efficacy of PTE without DHCA.

\section{Methodology}

\subsection{Study Design}

Approval from the institute Ethics \& research committee was obtained for this retrospective study. This study included 15 patients who underwent PTE for CTEPH, from July 2009 till September 2018, in the department of Cardiovascular \& Thoracic Surgery, All India Institute of Medical Sciences, New Delhi, India and records were reviewed. The patients were followed up with investigations like TTE and CTA were performed and the data were collected. Inclusion criteria laid down by American college of chest physicians were followed like 1) NYHA class III \& IV symptomatology, 2) Preoperative PVR $>300$ dynes $\cdot s \cdot \mathrm{cm}^{5}$, 3) Thrombus should be surgically accessible in main, lobar, segmental arteries, and 4) No debilitating co-morbidities. Accordingly our patients were selected following the inclusion criteria like those with diagnosed CTEPH, symptomaticon exertion and or at rest with surgically accessible thrombus in pulmonary arteries as delineated by CTA. We excluded all patients with acute pulmonary thromboembolism, harbouring infection/diagnosed sepsis, having other associated co-morbidities, the patients not fit to undergo CPB and those for emergency PTE. Apart from 
demographic variables, pulmonary artery pressures (systolic, diastolic and mean), central venous pressure (CVP), oxygen saturation (SpO2), CPB time, location of thrombus, postoperative course and any postoperative complications were studied. Pulmonary artery pressures (PAP) were measured by preop echocardiogram, intraoperatively before and after PTE by direct puncture transducing of main pulmonary artery (MPA), CVP and SpO2 measured before and after PTE. Location of thrombus was described by Jamieson's classification.

\subsection{Surgical Technique}

Following midline sternotomy and pericardiotomy, pulmonary artery pressure (PAP) was measured by direct puncture and transducing the MPA. Aortic and bicaval cannulation were done, total $\mathrm{CPB}$ was established, after achieving target activated clotting time $(\mathrm{ACT})>480$ seconds. Patient was not cooled, hence normothermia was maintained and heart was beating throughout the procedure. After making the main and branch pulmonary arteries free, an incision was made in the MPA at the level of bifurcation and extended towards right pulmonary artery (RPA). The pulmonary arteries were approached intrapericardially, bilateral pleurae were kept intact. The arteriotomy in the RPA was extended (if needed) laterally towards the lobar branch level; sump sucker was placed inside the RPA to tackle the bleed from the bronchial arteries. Another sucker was used to suck out the blood from left pulmonary artery (LPA). If there was any fresh loose thrombus, it was removed first as it could occlude the view as well as may dislodge and embolise distally. The plane of thromboendarterectomy was developed cautiously with blunt dissection and was confirmed by the pearly white appearance and the ease of separation. The plane should not go too deep which can lead to perforation of the artery and should not be too superficial which can lead to inadequate clearance of thrombus leading to persistent pulmonary hypertension. By gentle dissection, traction and counter traction, the thrombus was mobilised in the RPA, then further mobilization was done into the lobar and segmental levels by the same method of traction and counter traction. It should be made sure that, the distal most thrombus was not cut free but it was "tailed off", so that there was nothrombus left behind to avoid the distal arteriopathy. In case of difficulty in exposure, superior vena cava (SVC) could be transected so that adequate exposure of whole RPA with good exposure till lobar branch level could be attained. SVC was re-anastomosed once PTE was done by 5 - 0 polypropylene suture. Now the incision in MPA was extended to LPA and thrombus was removed in the same way as described for RPA above. Successful thromboendarterectomy was assured by the brisk bright red back bleed from the distal segments. Once thrombus was removed, the arteriotomy was closed after deairing the right heart and pulmonary arteries. Now the patient was weaned off CPB and then PAP was measured. Following this, protamine was administered and routine closure was done. Patients were shifted to the cardiac surgical ICU, hemodynamic parameters were monitored and mechanical ventilation done with 
lower tidal volume with peak end expiratory pressure (PEEP) \& at minimum FiO2 that maintained the $\mathrm{PaO} 270 \mathrm{mmHg}$ and $\mathrm{SpO} 2>90 \%-95 \%$. Usually they were electively ventilated for 24 hours for stabilizing their hemodynamics and pulmonary gas exchange. TTE was done to assess RV function and PA pressure from the Tricuspid Regurgitation (TR) jet velocity before weaning trial. Postoperatively diuretics (frusemide), antiplatelets (aspirin), oral anti-coagulants (warfarin) and pulmonary vasodilators were started to all the patients. PT-INR was maintained between 2 and 3. Before discharge, all the patients were evaluated by TTE and CTA.

Statistical analysis was done by SPSS version stata 14.1. The values were expressed as mean, mean \pm standard deviation or in numbers (\%). Paired $t$ test was used to compare preoperative and postoperative parameters. P Value $<0.05$ was considered statistically significant.

\section{Results}

Data of 15 patients were analysed. The mean age was 35.28 years and 10 of them were males, 5 were females. 10 patients (67\%) had a past history of deep vein thrombosis (DVT), treated medically and were on oral anticoagulation medications, and were stopped 5 days before surgery. The patients presented with major symptoms like dyspnoea on exertion (DOE), palpitations, cough, pedal edema and syncope. DOE was present in all 15 patients, palpitations in 5 patients and syncope in 4 patients. DOE was of NYHA class II in 6 patients (40\%), class III in 7 patients (47\%) and class IV in 2 patients (13\%). 2 patients (13\%) were admitted to intensive care unit preoperatively for management of congestive heart failure. Demographic and symptomatology details are illustrated in Table 1. One patient who had pulmonary sarcoidosis, developed deep venous thrombosis (DVT), a known complication [9], was very symptomatic (DOE NYHA class III), medically stabilised before surgery and another one had pulmonary Koch's disease. Right ventricular function, tricuspid valve regurgitation, pulmonary artery pressure were assessed by TTE in all patients. CTA revealed the extent of thrombus, status of proximal pulmonary arteries and the whole pulmonary vascular tree (Figure 1(a)). Table 2 depicts the intra-operative patient variables. The mean $\mathrm{CPB}$ time was $62.5 \pm 19.5$ minutes. Location of thrombus, as per Jamieson classification, type I was in 2 (13\%), type II was in $13(87 \%)$ patients and none in type III and type IV. Table 3 shows the pre \& post operative hemodynamic parameters. The postoperative pulmonary artery (PA) pressures (systolic, diastolic \& mean) and CVP were significantly lower compared to the preoperative values. The postoperative pulmonary artery pressures (systolic, diastolic \& mean) were significantly lower compared to the preoperative values $(\mathrm{mmHg})(73.35 \pm 14.12,19.28 \pm 8.60,36.50 \pm 11.30$ vs. $35.21 \pm 7.89,12.85 \pm$ $7.26,20.21 \pm 7.19$ respectively). The mean preop and postop CVP was $17.92 \pm$ $2.43 \mathrm{mmHg}$ and $11.78 \pm 1.92 \mathrm{mmHg}$ respectively, also statistically significant. The preop and postop oxygen saturation was $88.14 \% \pm 2.24 \%$ and $96.07 \% \pm$ 
Table 1. Demographic variables and preoperative data.

\begin{tabular}{lc}
\hline \multicolumn{1}{c}{ Variables } & Numbers \\
\hline Age (mean years) & 35.28 \\
Sex & 10 \\
- Male & 5 \\
- Female & 1.72 \\
BSA (m²) & 10 \\
DVT history & 15 \\
DOE & Nil \\
- Class I & 6 \\
- Class II & 7 \\
- Class III & 2 \\
- Class IV & 5 \\
Palpitations & 4 \\
Syncope & \\
Preoperative ICU admission (anti-failure & 2 \\
management) & \\
Associated morbidities & \\
- Diabetes mellitus & $2(14 \%)$ \\
- Smoking & $1(7 \%)$ \\
- Tuberculosis & $1(7 \%)$ \\
- Sarcoidosis &
\end{tabular}

Table 2. Intraoperative data.

\begin{tabular}{cc}
\hline Cardiopulmonary bypass time (minutes) & $62.5 \pm 19.5$ \\
\hline Jamieson classification & $2(13 \%)$ \\
Type I & $13(87 \%)$ \\
Type II & 0 \\
Type III & 0 \\
Type IV & 0 \\
\hline
\end{tabular}

Table 3. Comparison of preoperative and postoperative hemodynamic parameters.

\begin{tabular}{cccc}
\hline Parameters & Preoperative & Postoperative & Significance \\
\hline PAP systolic (mmHg) & $73.35 \pm 14.12$ & $35.21 \pm 7.89$ & $\mathrm{p}<0.05$ \\
PAP diastolic $(\mathrm{mmHg})$ & $19.28 \pm 8.60$ & $12.85 \pm 7.26$ & $\mathrm{p}<0.05$ \\
PA mean $(\mathrm{mmHg})$ & $36.50 \pm 11.30$ & $20.21 \pm 7.19$ & $\mathrm{p}<0.05$ \\
CVP (mmHg) & $17.92 \pm 2.43$ & $11.78 \pm 1.92$ & $\mathrm{p}<0.05$ \\
SpO2 $(\%)$ & $88.14 \pm 2.24$ & $96.07 \pm 2.01$ & $\mathrm{p}<0.05$ \\
\hline
\end{tabular}

PAP_Pulmonary Artery Pressure; CVP_Central Venous Pressure; SpO2-Oxygen Saturation.

$2.01 \%$, statistically significant. Postoperative TTE showed marked decrease in the right ventricular dimensions, decrease in the pulmonaryartery pressures and improved RV function. Figure 1(b) shows the postoperative CT angiogram where the contrast filling is seen in all the branch, segmental and subsegmental 


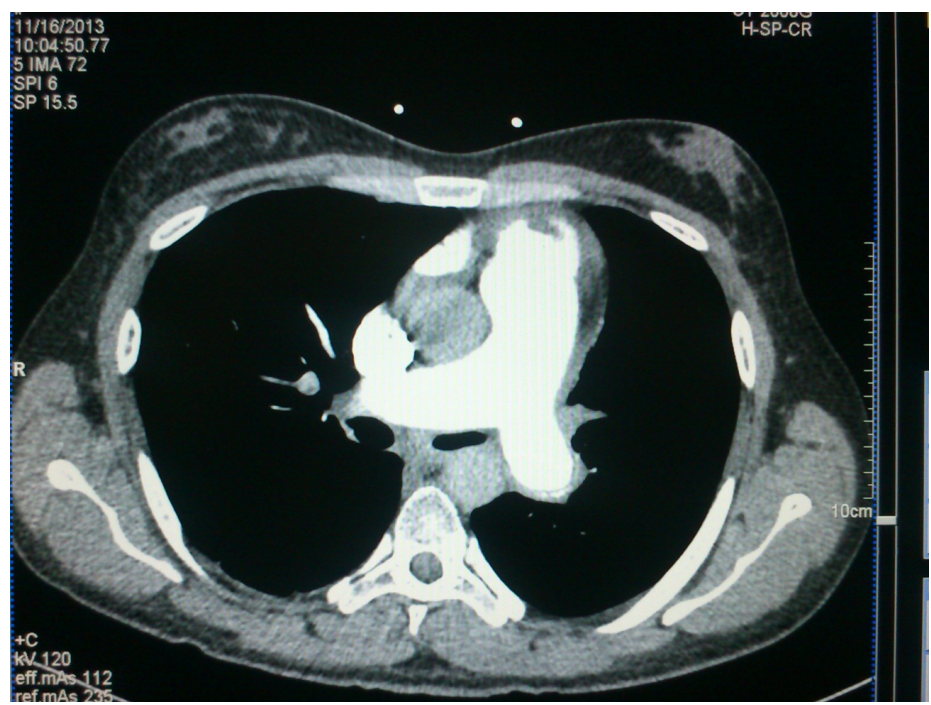

(a)

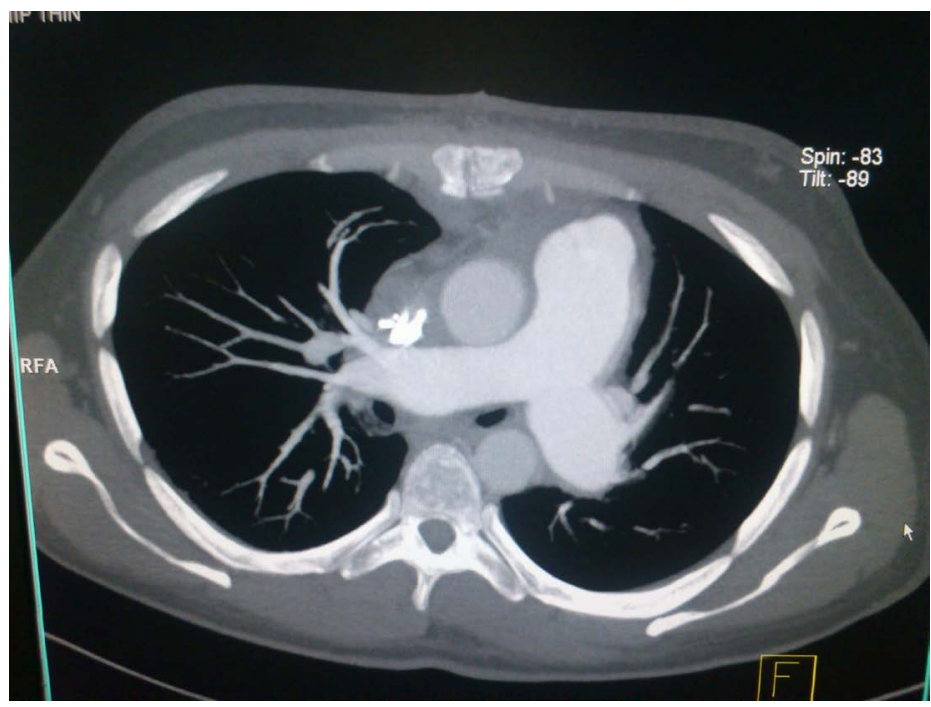

(b)

Figure 1. (a) Axial cut of CT angiogram showing cut off of branch pulmonary arteries and multiple filling defects; (b) Complete revascularisation of entire pulmonary arterial tree till segmental level post pulmonary thromboendarterectomy.

arteries with no residual thrombus and Figure 2 shows the resected specimen of the pulmonary thromboendarterectomy specimen of the right pulmonary artery involving lobar, segmental and subsegmental vessels. Note the tailed ending of the thrombus distally. The postoperative results were detailed in Table 4. The mean duration of ventilation was 34.14 hours. One patient (7\%) had difficulty in weaning from mechanical ventilation so underwent tracheostomy. Vasoactive Inotropic score (VIS) was calculated in the first 24 hours, the mean score was 10.91 \pm 4.7 (range of 10 to 26). Most of the patients required 2 inotropes and vasodilators during the postoperative course. The mean duration of ICU stay was 10.07 days.

Two patients required re-exploration, one for bleeding and another patient for pericardial effusion. Culture proven sepsis was identified in 2 patients. And both 


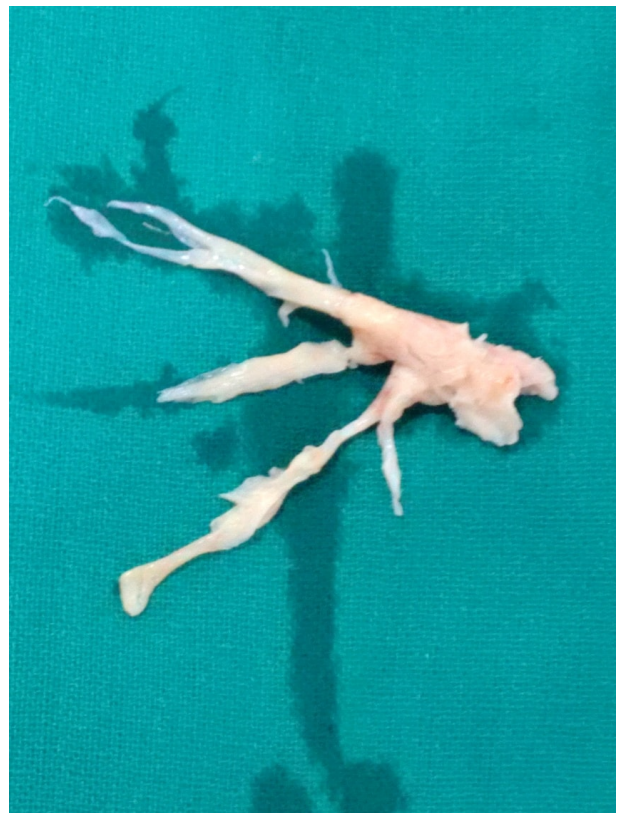

Figure 2. The resected specimen of the pulmonary thromboendarterectomy specimen of the right pulmonary artery involving lobar, segmental and subsegmental vessels. Note the tailed off ending of the thrombus distally.

Table 4. Postoperative results.

\begin{tabular}{cc}
\hline Duration of ventilation(hours) & 34.14 \\
Tracheostomy & $1(7 \%)$ \\
Vasoactive inotropic score & $10.91 \pm 4.7$ \\
Mean ICU stay (days) & 10.07 \\
Reexploration & $2(13 \%)$ \\
Pericardial effusion & $1(7 \%)$ \\
Sepsis & $2(14 \%)$ \\
Surgical site infection & Nil \\
Pulmonary hemorrhage & $1(7 \%)$ \\
ARDS & $1(7 \%)$ \\
Mortality & $1(7 \%)$ \\
Mean hospital stay (days) & 22.18 \\
Mean follow-up (months) & 36.46 \\
Quality of life & $13(87 \%)$ \\
NYHA I & $2(13 \%)$ \\
NYHA II & Nil \\
NYHA III, IV & \\
\hline & \\
\hline &
\end{tabular}

of them required prolonged mechanical ventilation, one of them was tracheostomised. Escherichia coli was isolated from tracheal aspirate from the tracheostomised patient. Broncho Alveolar Lavage (BAL) sample in the second patient yielded Acinetobacter and candida. Both the patients were treated successfully 
and recovered. Intra Pulmonary haemorrhage occurred in one patient in the immediate postoperative period, which was managed conservatively by mechanical ventilation with low tidal volume and high Positive End Expiratory Pressure (PEEP), and medical measures (fresh frozen plasma, platelet concentrate, tranexamic acid and protamine). Bleeding decreased from 9th postoperative day onwards and was successfully extubated on 13th postoperative day [9]. One of the patient had failed extubation, developed features of Acute Respiratory Distress Syndrome (ARDS) on postoperative day 5, elective tracheostomy was done for the ease of ventilation and lung toileting, managed conservatively. She was successfully weaned from ventilator on postoperative day 64 and her trachea was decannulated on postoperative day 90 . One patient died on 9th postoperative day, due to pneumonia, and respiratory failure. This patient had sarcoidosis, who underwent PTE uneventfully, had been extubated on $1^{\text {st }}$ postoperative day, then got reintubated on 4th postoperative day due to respiratory failure. He had developed pneumonia, septicemia and expired on 9th postoperative day. The mean hospital stay was 22.18 days. The shortest follow-up was 1 month and longest was 7 years. On follow-up the quality of life was better compared to preoperative period, 13 (87\%) patients were in NYHA class I and 2 (13\%) patients were in NYHA class II.

\section{Discussion}

Pulmonary thromboendarterectomy (PTE) remains the standard treatment for CTEPH since the time it was first published by University College of San Diego in the late 1980s [10]. PTE is considered safe and the technique has evolved since it has been first performed [11]. PTE under CPB \& DHCA is considered the standard technique till now because it prevents the bronchial back bleeding (caused by chronic hypoxia induced bronchial arterial hyperplasia) and helps complete removal of the thrombus from the pulmonary vascular bed [12]. DHCA, although helps in complete removal of the thrombus, but not without the dangers of potential neurological complications. It is becoming clear from many studies involving human body organ functioning, that oxygen consumption is never near zero even if the core temperature is reduced to $0^{\circ} \mathrm{C}$. This suggests that, some metabolic activity still go on irrespective of the temperature of cell, so the time limit of circulatory arrest should be accurate. Usually at a temperature of $18^{\circ} \mathrm{C}$, a safe circulatory arrest time period of 30 minutes is possible without any structural or functional derangement of brain functions [13] [14]. The temperature of brain is indirectly monitored from tympanic or nasopharyngeal temperature probes, hence this may not be so accurate. The brain protection in DHCA and the definite cut off time of circulatory arrest is not accurately predictable; hence there always remains a possibility of neurological injury irrespective of the different strategies of temperature \& blood gas management during DHCA. Several studies were suggestive of significant postoperative neurocognitive dysfunction following DHCA, with increasing age a risk factor for 
the same [15] [16]. None of our patient had any temporary or permanent neuro-cognitive dysfunction postoperatively. Apart from these, the opportunity to ensure the completeness of the procedure by brisk bright red-back bleed from the distal segmental vessels is not there if done under DHCA.

To avoid the above complications of DHCA, as described several attempts like antegrade cerebral perfusion, DTA occlusion by inflatable balloon catheter, double venting of left heart were made worldwide. All our 15 patients underwent PTE with normothermic beating heart CPB successfully without the need for DHCA which avoids the much debated risks of brain injury and long term neurologic sequelae.

Reperfusion pulmonary edema (RPE) is a specific immediate complication following PTE under DHCA. It is a high permeability edema, gives rise to difficult ventilation, high airway pressure and acute hypoxemia. The incidence is around $9.6 \%$ in a large European registry [17]. Usually, it develops 6 hours after surgery, which could be managed with deep sedation, aggressive diuretics, steroids, controlled Intermittent Positive Pressure Ventilation (IPPV) with high positive end expiratory pressure (PEEP) and at last extracorporeal membrane oxygenation (ECMO) support. By avoiding DHCA, the possibility of reperfusion injury to the lung parenchyma is minimized as at no point of time lung parenchyma is devoid of blood supply from bronchial circulation. Hence in our patients there was no RPE.

Intra-pulmonary haemorrhage is another rare complication with a reported incidence of $2 \%$, could happen within first 24 hours after PTE. One of our patients developed severe intrapulmonary haemorrhage within 8 hours of surgery and was managed by high controlled IPPV with PEEP, correcting coagulopathy, topical vasoconstrictors, reducing pulmonary artery pressures and maintaining haematocrit \& hemodynamic stability [18].

The perioperative mortality rate following PTE is around 4\% - 23\% [2] [11]. Patients with hemodynamic instability and high PVR $>1100$ dyne $\cdot s \cdot \mathrm{cm}^{-5}$ or mPAP > $50 \mathrm{mmHg}$ are predictors of perioperative mortality [19]. In our study, one patient (7\%) who had sarcoidosis, preoperative ICU admission for heart failure \& pulmonary edema, died on postoperative day 4 due to pneumonia, ARDS \& respiratory failure. The mortality rate was comparable to other studies of PTE.

The only major factor in predicting the longterm outcome is location \& extent of the thrombus in the pulmonary vascular tree found introperatively. Jamieson classified the disease (CTEPH) based on the intraoperative location of the thrombus. Type I is major vessel clot readily visible on opening pulmonary arteries, Type II is thickened intima, endarterectomy plane is created in main, lobar, segmental vessels. Type III is distal disease confined to segmental and subsegmental vessels and type IV is intrinsic small vessel disease [20]. Type I, II and to some extent type III are amenable to surgery. In type IV disease, surgery is not advised, because of poor results. 13 of our patients belong to type II and 2 of the were having type I pattern of disease and none was in type III or IV. 
The efficacy of the procedure was assessed intraoperative as well as postoperatively. Intraoperatively, by ensuring the complete removal of the thrombus, brisk back bleed and a resultant drop in the peak systolic and mean pulmonary arterial pressure after the thromboendarterectomy. Postoperatively, CTA did not show any residual thrombus in the pulmonary tree till segmental pulmonary artery level. On follow-up TTE marked decrease in right ventricular size and volume, decrease in tricuspid regurgitation, decrease in PAP were noted. This improvement in the pulmonary vascular hemodynamics and the reverse remodelling of right ventricle are the probable reasons for the clinical improvement and the improved quality of life following PTE [12] [21].

The 5 year survival of a patient with diagnosed CTEPH, with mPAP $>40$ $\mathrm{mmHg}$ is $30 \%$ and mPAP $>50 \mathrm{mmHg}$ was $10 \%$ [22] [23] [24]. Following a standard PTE, 5 year survival was around 90\%, compared to post lung transplantation 5 year survival of 50\% [25]. Hence, PTE definitely improves the quality of life and survival of patients with CTEPH. Our technique of performing PTE also yielded safe, effective, satisfactory results in the immediate postoperative period, with a complication free quality of life and with a low mortality rate at a maximum follow-up of up to 82 months, which was comparable to the results of other studies.

\section{Conclusion}

Pulmonary thromboendarterectomy under normothermic beating heart cardiopulmonary bypass has good immediate postoperative results with significant progressive improvement in hemodynamics and quality of life during the course of follow-up. The results were not only comparable to those of the procedure done under deep hypothermic circulatory arrest by other centres but also without its associated adverse events. This technique requires more expertise but gives equivalent good results in immediate and short- to mid-term follow-up with less morbidity than the standard procedure, but it requires long term follow-up to substantiate the evidence.

\section{Limitations}

The major limitation of the study is retrospective study with small number of patients and lack of long-term follow-up.

\section{Funds}

No fund was received from anybody.

\section{Acknowledgements}

We thank our patients and institutional support for this study.

\section{What Is Already Known?}

Pulmonary thromboendarterectomy under DHCA is the gold standard surgical 
treatment for CTEPH.

\section{What Does This Study Adds?}

Normothermic beating heart pulmonary thromboendarterectomy is a good alternative to that done under DHCA and also avoids the potential adverse effects of DHCA.

\section{Conflicts of Interest}

The authors declare no conflicts of interest regarding the publication of this paper.

\section{References}

[1] Pengo, V., Lensing, A.W., Prins, M.H., et al. (2004) Thromboembolic Pulmonary Hypertension Study Group. Incidence of Chronic Thromboembolic Pulmonary Hypertension after Pulmonary Embolism. The New England Journal of Medicine, 350, 2257-2264. https://doi.org/10.1056/NEJMoa032274

[2] Moser, K.M., Auger, W.R. and Fedullo, P.F. (1990) Chronic Major-Vessel Thromboembolic Pulmonary Hypertension. Circulation, 81, 1735-1743. https://doi.org/10.1161/01.CIR.81.6.1735

[3] Moser, K.M., Daily, P.O., Peterson, K., et al. (1987) Thromboendarterectomy for Chronic, Major-Vessel Thromboembolic Pulmonary Hypertension. Immediate and Long-Term Results in 42 patients. Annals of Internal Medicine, 107, 560-565.

https://doi.org/10.7326/0003-4819-107-4-560

[4] Pepke-Zaba, J., Delcroix, M., Lang, I., et al. (2011) Chronic Thromboembolic Pulmonary Hypertension (CTEPH): Results from an International Prospective Registry. Circulation, 124, 1973-1981. https://doi.org/10.1161/CIRCULATIONAHA.110.015008

[5] Masuda, M., Mogi, K., Nakaya, M., et al. (2001) Surgical Treatment for Chronic Pulmonary Thromboembolism under Cardiopulmonary Bypass with Selective Cerebral Perfusion. Surgery Today, 31, 108-112. https://doi.org/10.1007/s005950170192

[6] Hagl, C., Khaladj, N., Peters, T., et al. (2003) Technical Advances of Pulmonary Thromboendarterectomy for Chronic Thromboembolic Pulmonary Hypertension. European Journal of Cardio-Thoracic Surgery, 23, 776-781. https://doi.org/10.1016/S1010-7940(03)00029-0

[7] Mikus, P.M., Dell, A.A., Pastore, S., et al. (2006) Pulmonary Endarterectomy: Is There an Alternative to Profound Hypothermia with Cardiocirculatory Arrest? European Journal of Cardio-Thoracic Surgery, 30, 563-565.

[8] Zund, G., Pretre, R., Niederhauser, U., et al. (1998) Improved Exposure of the Pulmonary Arteries for Thromboendarterectomy. The Annals of Thoracic Surgery, 66, 1821-1823.

[9] Ungprasert, P., Srivali, N., Wijarnpreecha, K., et al. (2015) Sarcoidosis and Risk of Venous Thromboembolism: A Systematic Review and Meta-Analysis. Sarcoidosis Vasculitis \& Diffuse Lung Diseases, 32, 182-187.

[10] Kenneth, M., Pat, O.D., Kirk, P., et al. (1987) Thromboendarterectomy for Chronic, Major-Vessel Thromboembolic Pulmonary Hypertension: Immediate and Long-Term Results in 42 Patients. Annals of Internal Medicine, 107, 560-564. 
https://doi.org/10.7326/0003-4819-107-4-560

[11] Jamieson, S.W., Kapelanski, D.P., Sakakibara, N., et al. (2003) Pulmonary Endarterectomy: Experience and Lessons Learned in 1,500 Cases. The Annals of Thoracic Surgery, 76, 1457-1464. https://doi.org/10.1016/S0003-4975(03)00828-2

[12] Mayer, E. and Klepetko, W. (2006) Techniques and Outcomes of Pulmonary Endarterectomy for Chronic Thromboembolic Pulmonary Hypertension. Proceedings of the American Thoracic Society, 3, 589-593.

[13] Fisk, G.C., Wright, J.S., Hicks, R.G., et al. (1976) The Influence of Duration of Circulatory Arrest at 20 Degrees $\mathrm{C}$ on Cerebral Changes. Anaesth and Intensive Care, 4, 126-134.

[14] Fisk, G.C., Wright, J.S., Turner, B.B., et al. (1974) Cerebral Effects of Circulatory Arrest at 20 Degrees $\mathrm{c}$ in the Infant Pig. Anaesth and Intensive Care, 2, 33-42.

[15] Ergin, M.A., Galla, J.D., Lansman, S.L., et al. (1994) Hypothermic Circulatory Arrest in Operations on the Thoracic Aorta. Determinants of Operative Mortality and Neurologic Outcome. J Surg, 107, 788-797.

[16] Ergin, M.A., Griepp, E.B., Lansman, S.L., et al. (1994) Hypothermic Circulatory Arrest and Other Methods of Cerebral Protection during Operations on the Thoracic Aorta. Journal of Cardiac Surgery, 9, 525-537. https://doi.org/10.1111/j.1540-8191.1994.tb00886.x

[17] Mayer, E., Jenkins, D., Lindner, J., et al. (2011) Surgical Management and Outcome of Patients with Chronic Thromboembolicpulmonary Hypertension: Results from an International Prospective Registry. The Journal of Thoracic and Cardiovascular Surgery, 141, 702-710. https://doi.org/10.1016/j.jtcvs.2010.11.024

[18] Sahu, M., Kamath, N., Arun, S., et al. (2015) Successful Management of a Case of Severe Intrapulmonary Haemorrhage after Pulmonary Thrombo-Endarterectomy. Indian Journal of Thoracic and Cardiovascular Surgery, 31, 162-164. https://doi.org/10.1007/s12055-014-0312-y

[19] Hartz, R.S., Byrne, J.G., Levitsky, S., et al. (1996) Predictors of Mortality in Pulmonary Thromboendarterectomy. The Annals of Thoracic Surgery, 62, 1255-1260. https://doi.org/10.1016/0003-4975(96)00460-2

[20] Jamieson, S.W. and Kapelanski, D.P. (2000) Pulmonary Endarterectomy. Current Problems in Surgery, 37, 165-252. https://doi.org/10.1016/S0011-3840(00)80005-2

[21] Moser, K.M., Metersky, M.L., Auger, W.R., et al. (1993) Resolution of Vascular Steal after Pulmonary Thromboendarterectomy. Chest, 104, 1441-1444. https://doi.org/10.1378/chest.104.5.1441

[22] Moser, K.M. and Braunwauld, N.S. (1973) Successful Surgical Intervention in Severe Chronic Thromboembolic Pulmonary Hypertension. Chest, 64, 29-35. https://doi.org/10.1378/chest.64.1.29

[23] Dartvelle, P., Fadel, E., Mussot, S., et al. (2004) Chronic Thromboembolic Pulmonary Hypertension. European Respiratory Journal, 23, 637-648. https://doi.org/10.1183/09031936.04.00079704

[24] Lewczuk, J., Piszko, P., Jagas, J., et al. (2001) Prognostic Factors in Medically Treated Patients with Chronic Pulmonary Embolism. Chest, 119, 818-823. https://doi.org/10.1378/chest.119.3.818

[25] Freed, D.H., Thomson, B.M., Tsui, S.S., et al. (2008) Functional and Haemodynamic Outcome 1 Year after Pulmonary Thromboendarterectomy. European Journal of Cardio-Thoracic Surgery, 34, 525-529. https://doi.org/10.1016/j.ejcts.2008.04.018 